\title{
Is the MDS-UPDRS a Good Screening Tool for Detecting Sleep Problems and Daytime Sleepiness in Parkinson's Disease?
}

\author{
Krisztina Horváth, ${ }^{1}$ Zsuzsanna Aschermann, ${ }^{2}$ Péter Ács, ${ }^{2}$ Edit Bosnyák, ${ }^{2}$ Gabriella Deli, \\ Endre Pál, ${ }^{2}$ József Janszky, ${ }^{2,3}$ Béla Faludi, ${ }^{2}$ Ildikó Késmárki, ${ }^{4}$ Sámuel Komoly, ${ }^{2}$ \\ Magdolna Bokor, ${ }^{5}$ Eszter Rigó, ${ }^{5}$ Júlia Lajtos, ${ }^{6}$ Péter Klivényi, ${ }^{7}$ György Dibóo, ${ }^{7}$ \\ László Vécsei, ${ }^{78}$ Annamária Takáts, ${ }^{9}$ Adrián Tóth, ${ }^{9}$ Piroska Imre, ${ }^{10}$ Ferenc Nagy, ${ }^{11}$ \\ Mihály Herceg, ${ }^{11}$ Anita Kamondi, ${ }^{12}$ Eszter Hidasi, ${ }^{13}$ and Norbert Kovács ${ }^{2,3}$ \\ ${ }^{1}$ Doctoral School of Clinical Neuroscience, University of Pécs, Rét Utca 2, Pécs 7623, Hungary \\ ${ }^{2}$ Department of Neurology, University of Pécs, Rét Utca 2, Pécs 7633, Hungary \\ ${ }^{3}$ MTA-PTE Clinical Neuroimaging Research Group, Rét Utca 2, Pécs 7623, Hungary \\ ${ }^{4}$ Unified Health Centers of the City of Pécs, Dr. Veress Endre Utca 2, Pécs 7633, Hungary \\ ${ }^{5}$ Department of Neurology, Nyirő Gyula Hospital-National Institute of Psychiatry and Addictology, Dévai Utca 15a, Budapest, Hungary \\ ${ }^{6}$ Department of Neurology, Kenézy Gyula Hospital, Bartók Béla Utca 2-26, Debrecen, Hungary \\ ${ }^{7}$ Department of Neurology, University of Szeged, Semmelweis Utca 6, Szeged, Hungary \\ ${ }^{8}$ MTA-SZTE Neuroscience Research Group, Semmelweis Utca 6, Szeged, Hungary \\ ${ }^{9}$ Department of Neurology, Semmelweis University, Balassa Utca 6, Budapest, Hungary \\ ${ }^{10}$ Department of Neurology, Csolnoky Ferenc Hospital, Kórház Utca 1, Veszprém, Hungary \\ ${ }^{11}$ Department of Neurology, Kaposi Mór Hospital, Tallián Gyula Utca 20-32, Kaposvár 7400, Hungary \\ ${ }^{12}$ National Institute of Clinical Neuroscience, Amerikai Utca 57, Budapest, Hungary \\ ${ }^{13}$ Department of Neurology, University of Debrecen, Móricz Zsigmond Körút 22, Debrecen, Hungary
}

Correspondence should be addressed to Norbert Kovács; kovacsnorbert06@gmail.com

Received 14 August 2014; Accepted 15 October 2014; Published 17 November 2014

Academic Editor: Peter Hagell

Copyright (C) 2014 Krisztina Horváth et al. This is an open access article distributed under the Creative Commons Attribution License, which permits unrestricted use, distribution, and reproduction in any medium, provided the original work is properly cited.

\begin{abstract}
Movement Disorder Society-sponsored Unified Parkinson's Disease Rating Scale (MDS-UPDRS) has separate items for measuring sleep problems (item 1.7) and daytime sleepiness (1.8). The aim of our study was to evaluate the screening sensitivity and specificity of these items to the PD Sleep Scale 2nd version (PDSS-2) and Epworth Sleepiness Scale (ESS). In this nationwide, cross-sectional study 460 PD patients were enrolled. Spearman's rank correlation coefficients were calculated between the individual items, domains, and the total score of PDSS-2 and item 1.7 of MDS-UPDRS. Similarly, the items and the total score of ESS were contrasted to item 1.8 of MDS-UPDRS. After developing generalized ordinal logistic regression models, the transformed and observed scores were compared by Lin's Concordance Correlation Coefficient. Only item 3 difficulties staying asleep and the "disturbed sleep" domain of PDSS-2 showed high correlation with "sleep problems" item 1.7 of the MDS-UPDRS. Total score of PDSS-2 had moderate correlation with this MDS-UPRDS item. The total score of ESS showed the strongest, but still moderate, correlation with "daytime sleepiness" item 1.8 of MDS-UPDRS. As intended, the MDS-UPDRS serves as an effective screening tool for both sleep problems and daytime sleepiness and identifies subjects whose disabilities need further investigation.
\end{abstract}

\section{Introduction}

Recently the nonmotor symptoms (NMS) of Parkinson's disease (PD) have been increasingly recognized as major burden of quality of life $[1,2]$. The recently published Movement Disorder Society-sponsored Unified Parkinson's Disease Rating Scale (MDS-UPDRS) was developed to assess 
a wide variety of nonmotor symptoms with good scale properties. However, there are only a few published studies available contrasting the MDS-UPDRS to other validated scales on NMS. One of the papers demonstrated that the MDS-UPDRS Part I for nonmotor experiences of daily living had a strong convergent validity to the Non-Motor Symptoms Questionnaire with the exception of patients with the most severe NMS [1]. However, the NMS Questionnaire is a general scale and not an instrument specially designed for certain aspects of PD. In another recently published paper, the individual items of MDS-UPDRS Part I were validated against a number of scales for assessing individual NMS. However, this study included a relatively low number of patients $(n=94)$ and utilized the sole means of correlation calculations [3]. Although this research confirmed that the MDS-UPDRS Part I total score had a strong convergent validity with a composite score of scales for the NMS of PD and the majority of items of MDS-UPDRS Part I had at least moderate correlation with the representative nonmotor scales, the methodology applied was unable to confirm agreement and concordance. In our opinion, Spearman's correlation coefficient is an inappropriate measure of reliability because the strength of linear association, and not agreement, is measured. Therefore, it is possible to have a high degree of correlation even if agreement is poor $[4,5]$. Because one of the most important questions in assessing the model validity is if the modelpredicted values are precise and accurate at the same time [6], concordance correlation measurements should be utilized to establish agreement (concordance) by two scales.

Sleep-related problems are one of the most important and troublesome nonmotor aspects of PD. These problems are certainly multidimensional. For example, sleep-disturbances associated with PD might be equally due to troublesome nighttime OFF symptoms and other issues not specific for PD (e.g., microarousals caused by sleep apnea syndrome). Similarly, daytime sleepiness in PD might also be caused by $\mathrm{PD}$-specific or nonspecific causes.

MDS-UPDRS has separate items for measuring nighttime sleep-related problems (item 1.7) and daytime sleepiness (1.8) each ranging from 0 (not present) to 4 (severe) scores. The aim of our study was to evaluate the relationship of these items to the PD Sleep Scale 2nd version (PDSS-2) and the Epworth Sleepiness Scale (ESS).

The Parkinson's Disease Sleep Scale 2nd version is a validated scale to assess sleep problems in PD [7]. It is composed of 15 items evaluating three domains. Each item has a 5-point Likert-type scale ranging from 0 "never" to 4 "very often" (except for item 1 which is reversed). Each domain consists of clusters of five questions (motor symptoms at night: 4, 5, 6, 12, and 13; PD symptoms at night: 7, 9-11, and 15; and disturbed sleep: 1-3, 8, and 14) [7]. Symptoms on each domain can be scored in the range of $0-20$ points, higher scores meaning more nocturnal disturbance. The sum of the three domains gives the total score of PDSS-2 with the maximum value of 60 points. The threshold indicating sleep problems is 11 points for the Hungarian version of PDSS-2 [8-10].

Epworth Sleepiness Scale is composed of 8 items investigating a single domain to assess daytime sleepiness. The usefulness of ESS was demonstrated in PD by several studies
[11-14]. Each item is scaled from 0 "would never doze" to 3 "high chance to doze." The total score of ESS is the sum of all item scores (0-24 points), higher scores representing more severe daytime sleepiness. The cutoff value indicating daytime sleepiness in Hungarian PD patients is 5 points [8].

\section{Materials and Methods}

2.1. Patients. In this nationwide cross-sectional multicenter study 460 patients fulfilling the UK Brain Bank criteria for PD were enrolled. Each subject gave written consent in accordance with the ethical approval of the National Ethical Committee (184/2013. 14437/2013/EKU). Each patient was examined by a neurologist specialized in movement disorders. A portion of these patients (357/460) participated in the program of cultural adaptation and validation of the MDS-UPDRS into Hungarian. Data were summarized at University of Pécs (by KH and NK). Subsequently, levodopa equivalent dosage calculations were performed [15].

Patients were screened for the presence of dementia by the means of the Hungarian validated versions of Mattis Dementia Rating Scale [16] (MDRS, $n=345$ patients) and the Montreal Cognitive Assessment [17] (MOCA, $n=460$ patients). Patients receiving scores $\leq 125$ points on Mattis test and/or scores $\leq 22$ points on MOCA were considered as having major neurocognitive disorders [16].

2.2. Obtained Rating Scales. Besides sociodemographic and PD-historic data, the Hungarian validated versions of MDSUPDRS [18], PDSS-2 [8], and ESS [8, 19] were obtained. Because data from these scales were categorical, nonparametric tests were applied. As being part of the MDS-UPDRS, Hoehn-Yahr Scale was also used to detect the overall severity of PD. For description, medians with interquartile range (IR, 25th-75th percentiles) were calculated.

2.3. Prevalence and Correlations. Because the score of 0 on the items means symptom-free condition, the prevalence of each item was based on the portion of subjects having the score $>0$ point on that particular item.

For correlation, Spearman's rank correlation coefficients were calculated. For correlation coefficients, the values 00.299 were indicative of weak correlation, $0.300-0.599$ were indicative of moderate correlation, and $0.600-1.000$ were considered as high association [20].

\subsection{Regression Models and Lin's Concordance Correlation.} To evaluate the relationship between the scales, models of generalized ordinal logistic regression were built with bootstrap replication. Because most regression algorithms are very sample specific, their generalizability to other samples is quite limited. We chose a generalized logistic regression model with lasso regularization as this method allowed for bootstrap cross validation. Using this option we could generalize the results to samples outside the index sample.

To determine the relationship between the PDSS- 2 and the "sleep problems" item of MDS-UPDRS (1.7), generalized ordinal logistic regression was applied. First, we converted the 
PDSS-2 scores to MDS-UPDRS item 1.7 by regression analysis. Subsequently, Lin's Concordance Correlation Coefficient (LCCC) [21] was used to calculate the concordance between the true MDS-UPDRS scores and those estimated from the PDSS-2. The concordance was categorized by the value of LCCC as acceptable (0.601-0.900) and good (0.901-1.000).

Similar approach was utilized to determine the relationship between ESS and "daytime sleepiness" item of MDSUPDRS (1.8). Based on the obtained generalized ordinal logistic regression model we converted the ESS scores to MDS-UPDRS item 1.8 and subsequently LCCC were calculated between the true and estimated MDS-UPDRS 1.8 values.

2.5. Receiver Operating Characteristic (ROC) Curve. In order to analyze how efficiently the MDS-UPDRS differentiates patients having sleep-related problems from those who do not have any sleep-related problems, we applied ROC analysis. After categorization of patients into two groups based on their total score on PDDS-2 (patients with sleep problems: total PDSS-2 score $\geq 11$ points and patients without sleep problems: $<11$ points), we performed a second categorization based on MDS-UPDRS item 1.7 score (patients with sleep problems: $>0$ points, patients without sleep problems: 0 points). Subsequently these two different categorization methods were compared to obtain positive and negative predictive values, specificity, and sensitivity.

Afterwards, we also evaluated how appropriately the MDS-UPDRS 1.8 item identified patients with daytime sleepiness. Categorization based on the ESS scores (patients having daytime sleepiness: total score $\geq 5$ points versus those not having daytime sleepiness, ESS $<5$ points) was compared to that of MDS-UPDRS item 1.8.

2.6. Statistical Analysis. All statistical analyses were carried out using IBM SPSS software package (version 21, SPSS Inc., Chicago, USA). Statistical significance level was set to $5 \%$. Because the SPSS Suite did not have built-in functions for calculating LCCC, the freely available syntax files developed by Marta Garcia-Granero were utilized (available: http://gjyp.nl/marta/, accessed at Jan 7, 2013). Positive and negative predictive values were calculated by the utilization of the syntax adapted from the IBM website (http://www-01 .ibm.com/support/docview.wss?uid=swg21483380).

\section{Results}

3.1. Demographic and PD-Related Clinical Data. The subject population consisted of $460 \mathrm{PD}$ patients. The clinical characteristics are demonstrated in Table 1 . The LED was $214 \mathrm{mg}$ (14-324 mg, median and 25th-75th percentiles).

3.2. Sleep Problems. Based on MDS-UPDRS item 1.7, 315 patients $(68.4 \%)$ had sleep-related problems (prevalence, score $>0$ ). Only $1.7 \%$ of patients had the total score of 0 on PDSS-2. According to the PDSS total score, 324 patients $(70.4 \%)$ had sleep-related problems clinically (score $\geq 11$ points). Item 8 had the highest prevalence on PDSS2 (nocturia: $91.0 \%$ ), while the lowest was at item 7 (hallucinations: $15.0 \%)$. Out of the three domains of PDSS-2, the "disturbed sleep" had the highest prevalence (98.3\%). Prevalence, frequency, and median values of sleep-related items are demonstrated in Table 2.

Eight items of PDSS-2 had poor correlation with the "sleep problems" item of MDS-UPDRS (6: distressing dreams at night, 7: nighttime distressing hallucinations, 8: nocturia, 9: immobility at night, 11: nighttime muscle cramps in extremities, 12: painful posturing in the morning, 13: tremor, and 15: snoring or difficulties in breathing). Six items had moderate correlation with item 1.7 of MDS-UPDRS (1: bad sleep quality, 2: difficulties falling asleep, 4: restlessness of extremities at night, 5: urge to move extremities, 10: pain in extremities, and 14: tired and sleepy after waking in the morning). Furthermore, the total score and two domains of PDSS-2 (motor symptoms and PD symptoms at night) had also moderate correlation coefficients. Only a single item (3: difficulties staying asleep) and the "disturbed sleep" domain of PDSS-2 had high correlation with the "sleep problems" item of MDS-UPDRS (Table 2).

Generalized ordinal logistic regression model was developed for PDSS-2 items to estimate MDS-UPDRS item 1.7. Only PDSS items $1,2,3$, and 5 contributed significantly to the model (Supplementary Table 1 in Supplementary Materials available online at http://dx.doi.org/10.1155/2014/806169). The details of the ordinal logistic regression model are presented in Supplementary Table 2. The predicted MDSUPDRS 1.7 values were subsequently compared to the true MDS-UPDRS 1.7 values by LCCC. The value of LCCC was 0.833 (95\% confidence interval: $0.809-0.864$ ) demonstrating acceptable agreement between the PDSS-2 and the "sleep problems" item of MDS-UPDRS.

Based on the ROC analysis, MDS-UPDRS item 1.7 can differentiate between patients with and without sleep-related problems measured by PDSS-2 reliably (positive predictive value: 0.714 with the $95 \%$ CI of $0.633-0.794$; negative predictive value: 0.851 with the $95 \%$ CI of $0.809-0.887$; sensitivity: 0.754 with the $95 \%$ CI of $0.672-0.831$; and specificity: 0.827 with 95\% CI: 0.783-0.866).

3.3. Daytime Sleepiness. Based on MDS-UPDRS item 1.8, 345 patients $(75.0 \%)$ had problems with daytime sleepiness (prevalence, score $>0$ ). Only $4.74 \%$ of patients had the total score of 0 points on ESS. According to ESS total score, 312 patients (67.8\%) clinically had daytime sleepiness (ESS score $\geq 5$ points). None of the items of ESS showed high correlation with MDS-UPDRS item 1.8. While items 1-4 had moderate correlation, items 5-8 had only poor correlation. The highest value of correlation coefficient was observed between the total score of ESS and item 1.8 of MDS-UPDRS (0.494) (Table 3).

Generalized ordinal logistic regression model was developed for ESS items to estimate MDS-UPDRS item 1.8. Only ESS items 1, 2, 3, and 7 contributed significantly to the model (Supplementary Table 3). The details of the ordinal logistic regression model are presented in Supplementary Table 4. 
TABLE 1: Clinical characteristics of the study cohort.

\begin{tabular}{|c|c|c|}
\hline Characteristics & Mean/number & Percentage \\
\hline Age & $65.6 \pm 9.7$ & \\
\hline Disease-duration & $8.3 \pm 7.8$ & \\
\hline Education years & $11.8 \pm 3.2$ & \\
\hline Sex & 293 males & $63.7 \%$ \\
\hline Fluctuation & 236 & $51.3 \%$ \\
\hline Dyskinesia & 184 & $40.0 \%$ \\
\hline Hoehn-Yahr stage I & 31 & $6.7 \%$ \\
\hline Hoehn-Yahr stage II & 275 & $59.8 \%$ \\
\hline Hoehn-Yahr stage III & 115 & $25.0 \%$ \\
\hline Hoehn-Yahr stage IV & 28 & $6.1 \%$ \\
\hline Hoehn-Yahr stage V & 11 & $2.4 \%$ \\
\hline Deep brain stimulation therapy & 113 & $24.5 \%$ \\
\hline Levodopa/carbidopa intestinal gel infusion therapy & 10 & $2.2 \%$ \\
\hline Levodopa & 358 & $77.8 \%$ \\
\hline Dopamine agonists & 299 & $65.0 \%$ \\
\hline Without medication & 14 & $3.0 \%$ \\
\hline Levodopa and dopamine agonist combination & 118 & $25.7 \%$ \\
\hline COMT-inhibition & 208 & $45.2 \%$ \\
\hline MAO-inhibition & 108 & $23.5 \%$ \\
\hline Anticholinergic therapy & 10 & $2.2 \%$ \\
\hline Presence of dementia & 10 & $2.2 \%$ \\
\hline Antipsychotic medication & 8 & $1.74 \%$ \\
\hline Sedative medication usage & 25 & $5.43 \%$ \\
\hline
\end{tabular}

Presence of dementia was defined as having scores $\leq 125$ points on Mattis Dementia Rating Scale and/or scores $\leq 22$ points on Montreal Cognitive Assessment.

The predicted MDS-UPDRS 1.8 values were subsequently compared to the true MDS-UPDRS 1.8 values by LCCC. The value of LCCC was 0.638 (95\% confidence interval: 0.561$0.708)$ demonstrating acceptable agreement between ESS and "daytime sleepiness" item of MDS-UPDRS.

Based on the ROC analysis, MDS-UPDRS item 1.8 can differentiate between patients with and without daytime sleepiness measured by ESS reliably (positive predictive value: 0.617 with the $95 \%$ CI of $0.527-0.703$; negative predictive value: 0.777 with the $95 \%$ CI of $0.731-0.819$; sensitivity: 0.580 with the $95 \%$ CI of $0.500-0.660$; and specificity: 0.859 with 95\% CI: 0.817-0.895).

\section{Discussion}

Our data supports that the PDSS-2 has a strong and convergent validity to "sleep problems" item of MDS-UPDRS. Several items of disturbed sleep domain (items 1-3) had the best correlation with MDS-UPDRS item 1.7. This relationship is not surprising, because the question of item 1.7 queries the following: "Over the past week, have you had trouble going to sleep at night or staying asleep through the night? Consider how rested you felt after waking up in the morning." The possible answers in MDS-UPDRS emphasize the length of staying asleep (1: easily able to get a full night sleep, 2: with difficulties can achieve a full night sleep, 3: stay asleep more than the half the night, and 4: less than half of the night). The most concordant item (number 3) of PDSS-2 asks also about difficulty staying asleep, and the other acceptably concordant questions (1: bad sleep quality and 2: difficulties falling asleep) also contribute to this "disturbed sleep" domain.

For daytime sleepiness, we found considerably smaller correlation between the ESS and MDS-UPDRS item 1.8 than Gallagher and coworkers did [3]. In their paper Spearman's correlation coefficient was 0.56 based on the sample of 94 patients while our 0.494 value was based on the analysis of 460 patients. The discrepancy might be due to the differences in the sample size and other patient characteristics. We might assume at least two reasons behind these low correlation coefficients: MDS-UPDRS and ESS apply different timeframe and severity measurements. Whereas ESS asks the patient to base his/her answer referring to "recent times," the MDS-UPDRS assesses the usual function "over the past week including today." ESS evaluates how likely the patient will doze off in different situations (e.g., sitting and reading, being a passenger in a car, and sitting and talking to someone else). This type of severity scale assesses the frequency and likelihood to differentiate between scores 1-3. On the contrary, the severity scale of MDS-UPDRS item 1.8 is based not only on the frequency (e.g., 3: sometimes versus 4: often), but also on the different types of conditions where the patient would fall asleep (e.g., 2: being alone and relaxing; 3-4: eating or talking to people). Despite these concerns, we found acceptable agreement between the ESS items and the daytime sleepiness item of MDS-UPDRS. 


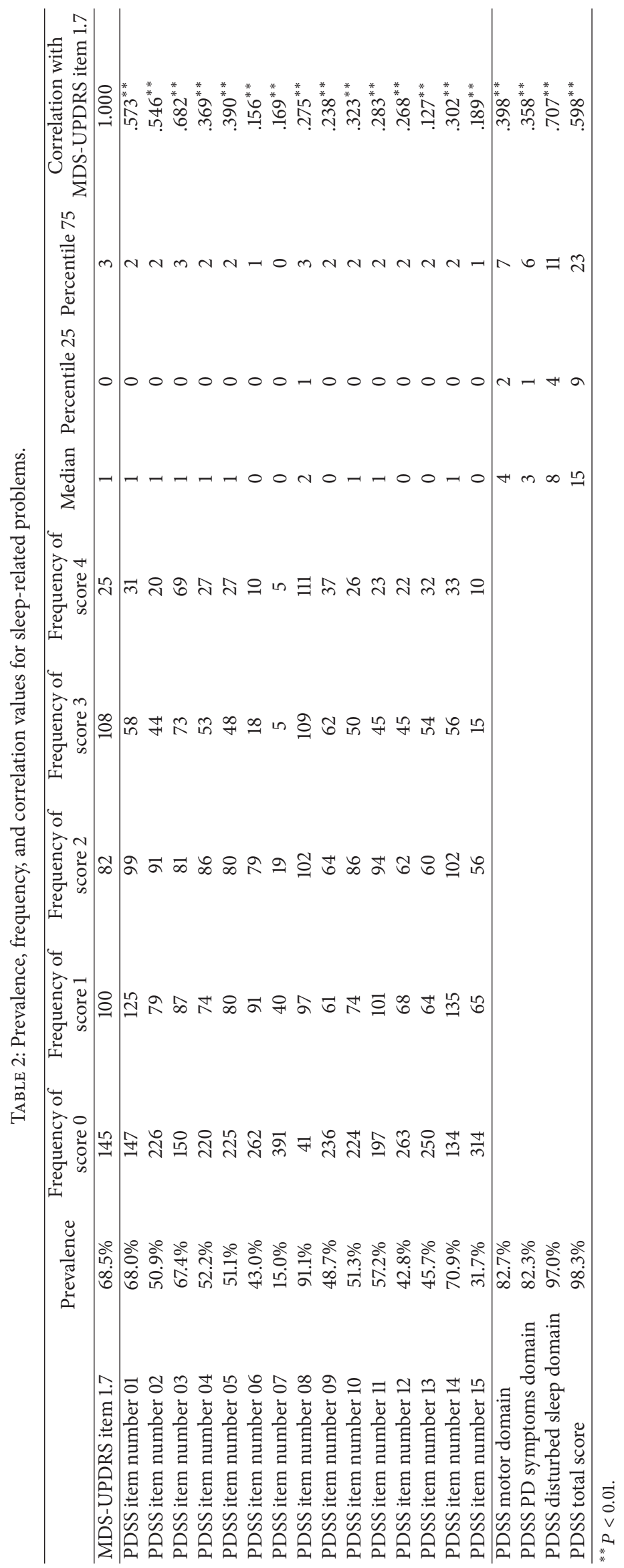




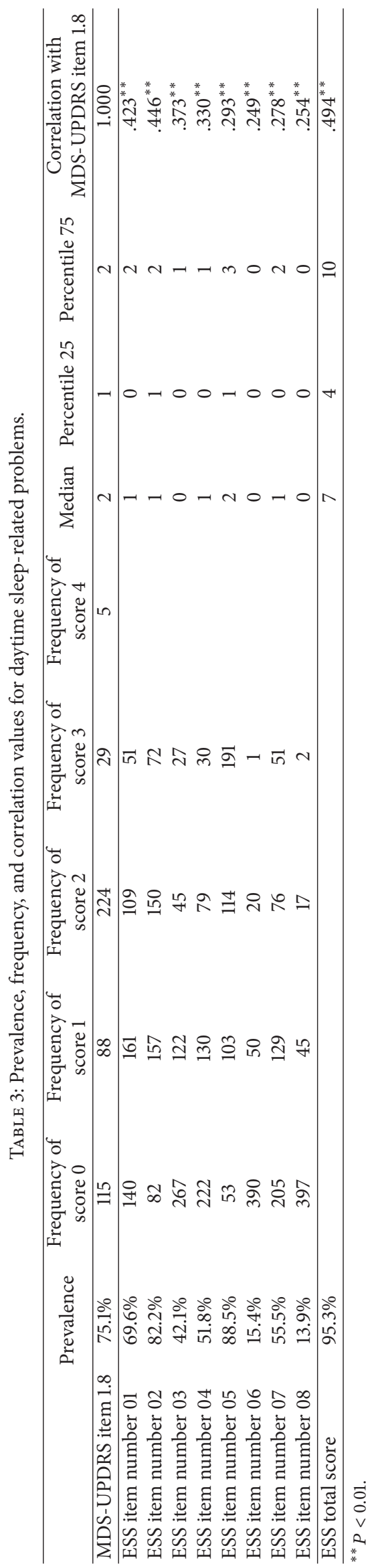


The authors are aware of some limitations of the study. Despite having a relatively large sample of patients, the majority of the subjects had mild to moderate disease severity (Hoehn-Yahr Scale: 1-3). To overcome this problem, we utilized a generalized logistic regression model with lasso regularization as this method allowed for bootstrap cross validation. Using this option we could generalize the results to samples outside the index sample.

\section{Conclusions}

Our data demonstrate that the MDS-UPDRS nonmotor experiences of daily works as a good screening questionnaire aimed at identifying areas of disability related to sleep problems and daytime sleepiness. The scale itself registers presence and severity of items but has an official APPENDIX with recommended more specific scales to pursue areas in more detail. As a screening tool, there are some aspects of sleep and daytime sleepiness which are not covered by MDSUPDRS, but the presence of nonzero scores on these MDSUPDRS items allows for fruitful utilization of more specific and detailed rating instruments. In our view, studies focusing on the sleep-related problems in PD can begin with the MDSUPDRS to accurately identify the cohort with sleep problems, and then this group can be studied with the PDDS-2 and ESS serving to assess all major dimensions in greater detail.

$\begin{array}{ll}\text { Abbreviations } \\ \text { ESS: } & \text { Epworth Sleepiness Scale } \\ \text { LCCC: } & \text { Lin's Concordance Correlation Coefficient } \\ \text { MDRS: } & \text { Mattis Dementia Rating Scale } \\ \text { MDS-UPDRS: } & \text { Movement Disorder Society-sponsored } \\ & \text { Unified Parkinson's Disease Rating Scale } \\ \text { MOCA: } & \text { Montreal Cognitive Assessment } \\ \text { NMS: } & \text { Nonmotor symptoms } \\ \text { PD: } & \text { Idiopathic Parkinson's disease } \\ \text { PDSS-2: } & \text { Parkinson's Disease Sleep Scale 2nd } \\ & \text { version } \\ \text { ROC: } & \text { Receiver Operating Characteristic. }\end{array}$

\section{Disclosure}

Krisztina Horváth reported no financial disclosure. Adrián Tóth reported no financial disclosure. Zsuzsanna Aschermann received $<1000$ EUR consultation fees from Hungarian subsidiaries of Novartis, GlaxoSmithKline, UCB, and Teva Pharmaceutical Industries Ltd. Regarding this study the author did not receive any corporate funding. Sámuel Komoly received $<1000$ EUR consultation fees from Hungarian subsidiaries of Biogen, TEVA, Astellas, Pfizer, and Novartis. Regarding this pilot study the author did not receive any corporate funding. Gabriella Deli reported no financial disclosure. Edit Bosnyák reported no financial disclosure. Péter Ács reported no financial disclosure. Réka horváth reported no financial disclosure. József Janszky received $<1000$ EUR consultation fees from Hungarian subsidiaries of UCB, GlaxoSmithKline, and Eisai. Regarding this pilot study the author did not receive any corporate funding. József Janszky is supported by the government-based Bolyai Scholarship of Hungarian Academy of Sciences. Norbert Kovács received $<1000$ EUR consultation fees from Hungarian subsidiaries of Medtronic, Boehringer Ingelheim, Novartis, GlaxoSmithKline, UCB, Krka, and Abbvie. Regarding this study the author did not receive any corporate funding. Péter Klivényi received $<1000$ EUR consultation fees from Hungarian subsidiaries of Boehringer Ingelheim, Novartis, GlaxoSmithKline, UCB, and Abbvie. Regarding this study the author did not receive any corporate funding. László Vécsei received $<1000$ EUR consultation fees from Hungarian subsidiaries of Biogen, TEVA, Richter, Pfizer, and Novartis. Regarding this pilot study the author did not receive any corporate funding.

\section{Conflict of Interests}

The authors declare that there is no conflict of interests regarding the publication of this paper.

\section{Authors' Contribution}

Krisztina Horváth contributed to conception, organization, and execution of research project; design, execution, review, and critique of statistical analysis; and writing of the first draft, review, and critique of the paper. Adrián Tóth contributed to execution of research project; review and critique of statistical analysis; and review and critique of the paper. Zsuzsanna Aschermann contributed to organization of research project; review and critique of statistical analysis; and review and critique of the paper. Sámuel Komoly contributed to organization of research project; review and critique of statistical analysis; and review and critique of the paper. Gabriella Deli contributed to organization of research project; review and critique of statistical analysis; and review and critique of the paper. Edit Bosnyák contributed to organization of research project; review and critique of statistical analysis; and review and critique of the paper. Péter Ács contributed to organization of research project; review and critique of statistical analysis; and review and critique of the paper. Réka Horváth contributed to organization of research project; review and critique of statistical analysis; and review and critique of the paper. József Janszky contributed to conception of research project; review and critique of statistical analysis; and review and critique of the paper. Norbert Kovács contributed to conception, organization, and execution of research project; design, execution, review, and critique of statistical analysis; and writing of the first draft, review, and critique of the paper.

\section{Acknowledgments}

The authors thank Drs. Christopher Goetz and Glenn Stebbins for their review of the data and assistance in preparing the paper prior to submission. Our study was supported 
by the Bolyai Scholarship of Hungarian Academy of Sciences, OTKA PD103964, AOK_OTKA_KA-2011, TÁMOP4.2.2.A-11/1/KONV-2012-0017, TÁMOP-4.2.2.A-11/1/KONV2012-0052, and Hungarian Brain Research Program Grant no. KTIA_13_NAP-A-II/10 government-based funds.

\section{References}

[1] D. Santos-García and R. de la Fuente-Fernández, "Impact of non-motor symptoms on health-related and perceived quality of life in Parkinson's disease," Journal of the Neurological Sciences, vol. 332, no. 1-2, pp. 136-140, 2013.

[2] P. Martinez-Martin, C. Rodriguez-Blazquez, M. M. Kurtis, and K. R. Chaudhuri, "The impact of non-motor symptoms on health-related quality of life of patients with Parkinson's disease," Movement Disorders, vol. 26, no. 3, pp. 399-406, 2011.

[3] D. A. Gallagher, C. G. Goetz, G. Stebbins, A. J. Lees, and A. Schrag, "Validation of the MDS-UPDRS part I for nonmotor symptoms in Parkinson's disease," Movement Disorders, vol. 27, no. 1, pp. 79-83, 2012.

[4] G. Rankin and M. Stokes, "Reliability of assessment tools in rehabilitation: an illustration of appropriate statistical analyses," Clinical Rehabilitation, vol. 12, no. 3, pp. 187-199, 1998.

[5] J. M. Bland and D. G. Altman, "Statistical methods for assessing agreement between two methods of clinical measurement," The Lancet, vol. 1, no. 8476, pp. 307-310, 1986.

[6] L. O. Tedeschi, "Assessment of the adequacy of mathematical models," Agricultural Systems, vol. 89, no. 2-3, pp. 225-247, 2006.

[7] C. Trenkwalder, R. Kohnen, B. Högl et al., "Parkinson's disease sleep scale-validation of the revised version PDSS-2," Movement Disorders, vol. 26, no. 4, pp. 644-652, 2011.

[8] N. Kovács, E. Pál, J. Janszky et al., "Parkinson's disease sleep scale-2 and Epworth sleep scale," Journal of the Neurological Sciences, vol. 333, p. e139, 2013.

[9] K. Horváth, Z. Aschermann, P. Ács et al., “Test-retest validity of Parkinson's disease sleep scale 2nd version (PDSS-2)," Journal of Parkinson's Disease, 2014.

[10] N. Kovács, Z. Aschermann, P. Ács et al., "The impact of levodopa-carbidopa intestinal gel on health-related quality of life in Parkinson's disease," Ideggyogyaszati Szemle, vol. 67, pp. 245-250, 2014.

[11] P. Hagell and J.-E. Broman, "Measurement properties and hierarchical item structure of the Epworth sleepiness scale in Parkinson's disease," Journal of Sleep Research, vol. 16, no. 1, pp. 102-109, 2007.

[12] S. Kumar, M. Bhatia, and M. Behari, "Excessive daytime sleepiness in Parkinson's disease as assessed by Epworth Sleepiness Scale (ESS)," Sleep Medicine, vol. 4, no. 4, pp. 339-342, 2003.

[13] I. Ghorayeb, A. Loundou, P. Auquier, Y. Dauvilliers, B. Bioulac, and F. Tison, "A nationwide survey of excessive daytime sleepiness in Parkinson's disease in France," Movement Disorders, vol. 22, no. 11, pp. 1567-1572, 2007.

[14] J. G. Goldman, R. A. Ghode, B. Ouyang, B. Bernard, C. G. Goetz, and G. T. Stebbins, "Dissociations among daytime sleepiness, nighttime sleep, and cognitive status in Parkinson's disease," Parkinsonism and Related Disorders, vol. 19, no. 9, pp. 806-811, 2013.

[15] C. L. Tomlinson, R. Stowe, S. Patel, C. Rick, R. Gray, and C. E. Clarke, "Systematic review of levodopa dose equivalency reporting in Parkinson's disease," Movement Disorders, vol. 25, no. 15 , pp. 2649-2653, 2010.
[16] B. Kaszás, N. Kovács, I. Balás et al., "Sensitivity and specificity of addenbrooke's cognitive examination, mattis dementia rating scale, frontal assessment battery and mini mental state examination for diagnosing dementia in Parkinson's disease," Parkinsonism and Related Disorders, vol. 18, no. 5, pp. 553-556, 2012.

[17] M. Volosin, K. Janacsek, and D. Németh, "Hungarian version of the Montreal Cognitive Assessment (MoCA) for screening mild cognitive impairment," Psychiatria Hungarica, vol. 28, no. 4, pp. 370-392, 2013.

[18] K. Horváth, Z. Aschermann, P. Ács et al., "Validation of the Hungarian MDS-UPDRS: why do we need a new Parkinson scale?" Ideggyogyaszati Szemle, vol. 67, no. 3-4, pp. 129-134, 2014.

[19] E. Pal, F. Nagy, Z. Aschermann, E. Balazs, and N. Kovacs, "The impact of left prefrontal repetitive transcranial magnetic stimulation on depression in Parkinson's disease: a randomized, double-blind, placebo-controlled study," Movement Disorders, vol. 25, no. 14, pp. 2311-2317, 2010.

[20] P. Martinez-Martin, K. R. Chaudhuri, J. M. Rojo-Abuin et al., "Assessing the non-motor symptoms of Parkinson's disease: MDS-UPDRS and NMS scale," European Journal of Neurology, 2013.

[21] L. I.-K. Lin, "A concordance correlation coefficient to evaluate reproducibility," Biometrics, vol. 45, no. 1, pp. 255-268, 1989. 


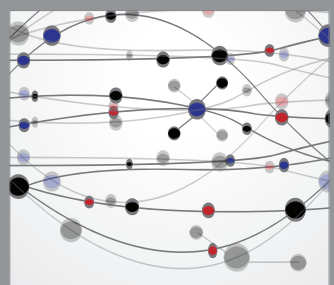

The Scientific World Journal
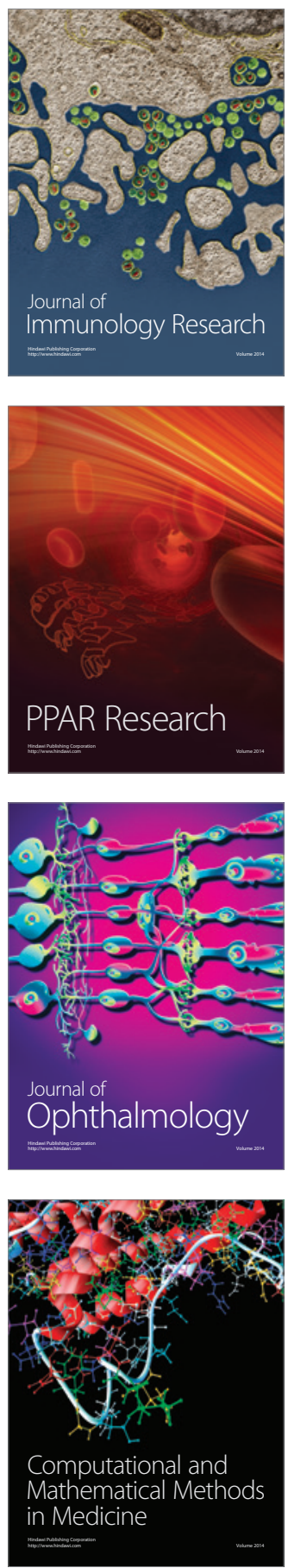

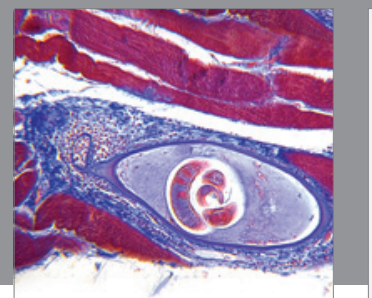

Gastroenterology

Research and Practice
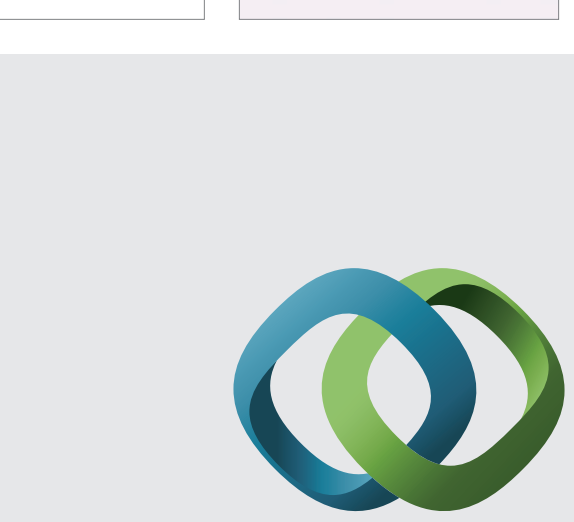

\section{Hindawi}

Submit your manuscripts at

http://www.hindawi.com
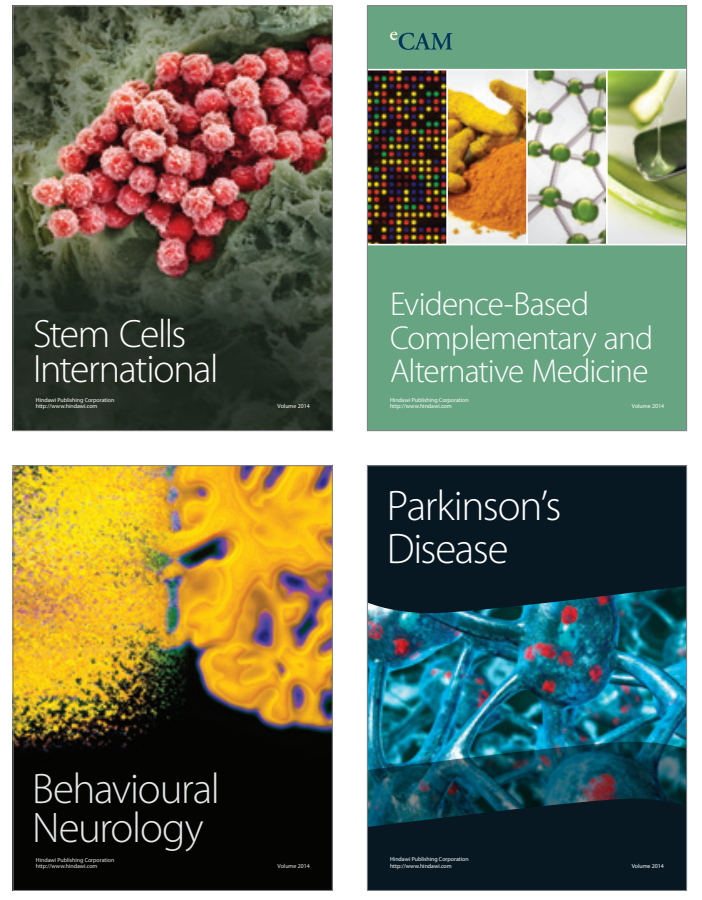
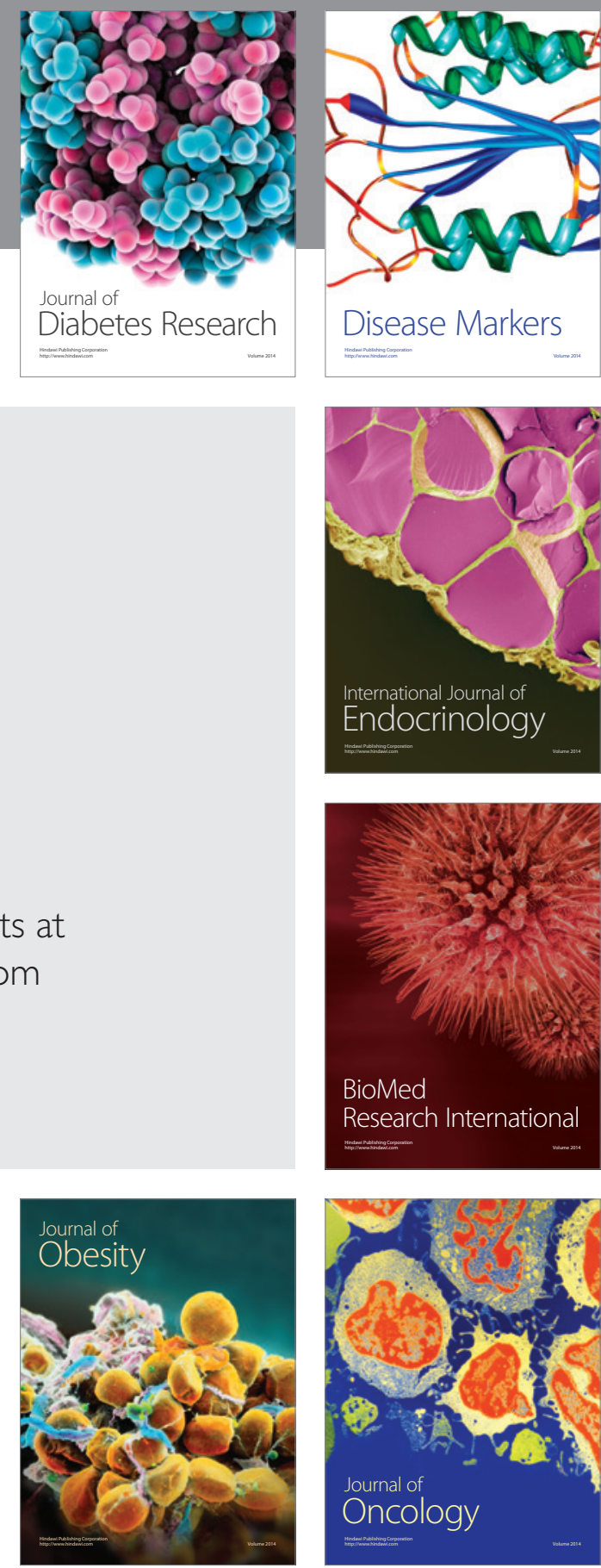

Disease Markers
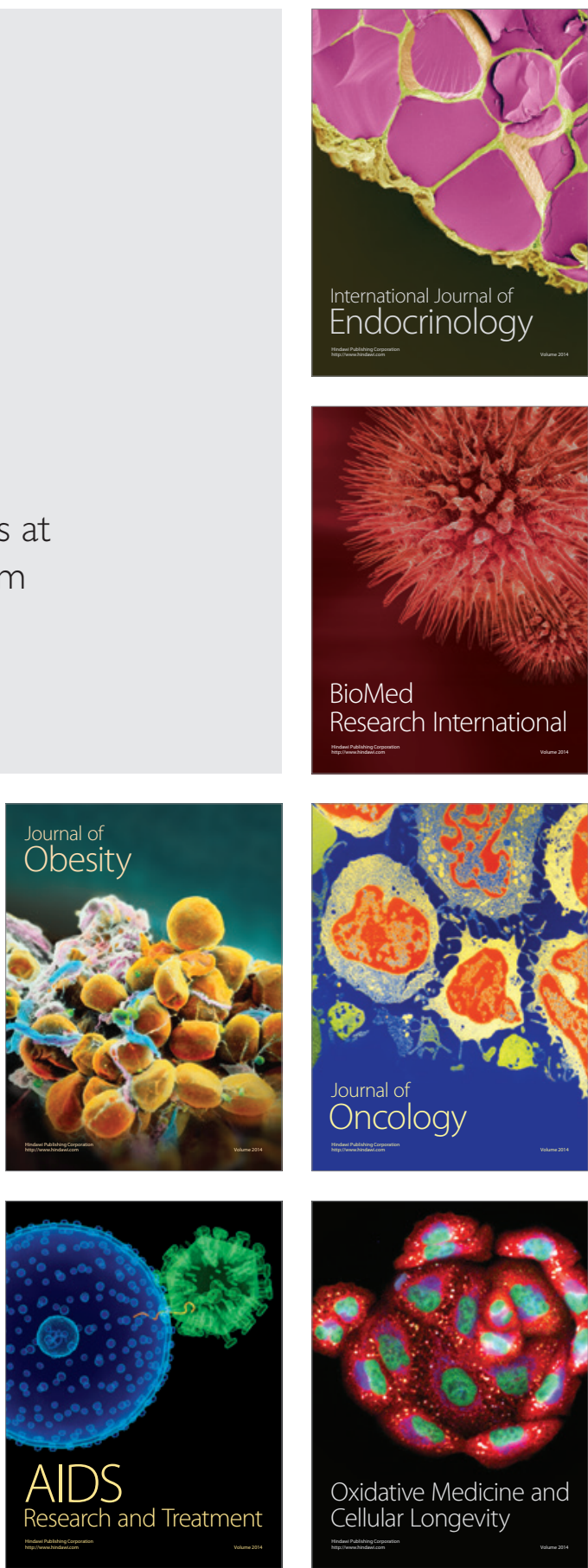